\title{
Automated analysis of platelet microstructures using a feature length orientation space
}

\author{
A. Campbell ${ }^{1, *}$ (1) P. Purray ${ }^{1}$, E. Yakushina ${ }^{1}$, A. Borocco $^{2}$, P. Dokladal ${ }^{2}$, E. Decencière ${ }^{2}$, \\ W. Ion ${ }^{1}$, and S. Marshall ${ }^{1}$ \\ ${ }^{1}$ University of Strathclyde, Glasgow, Scotland \\ ${ }^{2}$ Center for Mathematical Morphology, MINES Paris - PSL Research University, Fontainebleau, France
}

Received: 5 July 2021

Accepted: 12 October 2021

Published online:

3 January 2022

(C) The Author(s) 2021

\begin{abstract}
The ability to measure elongated structures such as platelets and colonies, is an important step in the microstructural analysis of many materials. Widely used techniques and standards require extensive manual interaction making them slow, laborious, difficult to repeat and prone to human error. Automated approaches have been proposed but often fail when analysing complex microstructures. This paper addresses these challenges by proposing a new, automated image analysis technique, to reliably assess platelet microstructure. Tools from Mathematical Morphology are designed to probe the image and map the response onto a new feature-length orientation space (FLOS). This enables automated measurement of key microstructural features such as platelet width, orientation, globular volume fraction, and colony size. The method has a wide field of view, low dependency on input parameters, and does not require prior thresholding, common in other automated analysis techniques. Multiple datasets of complex Titanium alloys were used to evaluate the new techniques which are shown to match measurements from expert materials scientists using recognized standards, while drastically reducing measurement time and ensuring repeatability. The per-pixel measurement style of the technique also allows for the generation of useful colourmaps, that aid further analysis and provide evidence to increase user confidence in the quantitative measurements.
\end{abstract}

Handling Editor: David Cann.

Address correspondence to E-mail: andrew.j.campbell@strath.ac.uk 


\section{Introduction}

Microstructural analysis is key to understanding material properties and is therefore important for quality assurance and optimization of manufacturing processes. Standardised analysis methods exist to enable skilled materials scientists to perform such analysis [1, 2] but the number and complexity of features to consider makes this process very slow and difficult to repeat. Materials such as the widely used Ti-6Al-4V alloy, have microstructures consisting of multiple distinct phases, with some phases being further subdivided into groupings of globular grains and fine elongated platelets, depending on material processing [3]. Accurate and robust analysis requires statistical measurements of the morphology of each of these features. Automated image processing methods can minimize the input and time required from domain experts, however, existing techniques are not ideal for platelet microstructures. For example, a recent technique has achieved reliable automated measurements of globular grains in microstructures [4], however, is not effective in measuring platelets. Platelets are very narrow in one dimension making them difficult to identify and easily obscured by noise. This problem is amplified when boundary delineation is poor, which is common in images of complex microstructures. A single microstructure may also contain multiple modalities of feature, such as globular grains and elongated platelets, which must be analyzed separately [5]. A set of image processing techniques specifically for analysing platelets has been published by Tiley et al. [6] and later extended by Collins et al. [7]. These provide the best current solution to the authors knowledge, however, only measurements of platelet width are fully automatic and these are only accurate for platelets with very high aspect ratios [7]. In this paper, we provide an analysis solution that can automatically measure a wide range of features including, platelet width, orientation, volume fraction of globular alpha, and colony size, where a colony is defined as a set of spatially clustered, parallel platelets. The proposed method is built using an image processing technique called mathematical morphology for feature extraction and mapping this to an orientation space, from which microstructural properties can be computed. We demonstrate our new technique on $\mathrm{Ti}-6 \mathrm{Al}-4 \mathrm{~V}$ as its complex microstructure is challenging for automated software to measure and contains a wide variety of features.

\section{Existing image analysis methods}

While the nuances of measuring platelets are challenging, useful theories for analyzing linear structures in images are well studied due to their prominence in fields such as remote sensing [8], medical imaging $[9,10]$ and manufacturing $[11,12]$. Historically, techniques such as the Hough Transform [13] and steerable filters [14] have success in measuring the orientation of both elongated and other [15] structures. However, these approaches do not measure object width and can produce errors when object shape and size are inconsistent. The Distance Transform [16] can facilitate width measurements, but gives no direct orientation or shape information. Adhikari et al. [17] recently presented a variation of this by diviging the area of objects by the length of the ridgelines of the Distance Transform, known as the Morphological Skeleton [18]. However, the area calculation requires an accurate initial detection of objects that is often not possible in complex microstructures. Very recent work by Merveille et al. [] address the limitations of such methods when studying curvilinear structures by using a novel operator called Ranking the Orientation Responses for Path Operators (RORPO). However, this approach is limited to extracting measurement of curvilinearity and orientation, rather than 8size.

Segmentation approaches are also extremely popular and allow properties of individual features to be measured [19, 20]. In recent years, machine learning methods, particularly those based on convolutional neural networks and deep learning have become popular for segmentation and other inspection problems [21-26]. While these techniques are highly successful in medical imaging [25] the more limited datasets available for most materials science problems make this hard to replicate here [26]. Such methods also often perform well on classifying features [23 24] but are less well suited to measuring individual instances of these features. This is illustrated in recent work which uses deep learning to accurately classify microstructure type but then requires post-processing from traditional image processing to measure each feature [27]. 
Approaches based on Mathematical Morphology appear best suited to analyse thin structures as measurement do not necessarily require prior segmentation. Statella et al. [28] demonstrated the use of granulometric techniques for width measurements of dust devil tracks on Mars. However, the techniques deployed a single shape of symmetrical structuring element (SE), so did not extract orientation and shape information. Conversely, Rivest et al. [29] measure only orientation by computing the morphological gradient from two perpendicular SEs, so does not obtain size information. Similarly, a recent paper from Borocco et al. [11] use such SE's to analyse elongated features in graphene but focus only on orientation. Borocco et al. show in [11] that this can be achieved effectively by first mapping the orientation measurement at each pixel onto a binary orientation space before applying a bespoke grouping algorithm to the data in this new domain. However, this technique requires a pre-determined SE parameters which are challenging to optimize effectively. The rotation of an SE also inherently provides some size and shape information that a binary orientation space can not exploit.

This paper presents a morphological approach using multivariate SEs to provide a robust solution that provides additional information and limits parametrisation dependency compared to existing techniques. By mapping the extracted information onto a feature length orientation space (FLOS) many useful microstructural features can be measured. The technique provides both quantitative measurements and useful visualisation to aid further analysis.

\section{Measurement using mathematical morphology}

Mathematical Morphology is a theory and image processing technique, first introduced by Matheron [30] and Serra [31]. It includes techniques which analyse spatial structures in images by probing an image with a predefined set, known as a structuring element (SE), B. This paper uses the Morphological Opening, $\varphi$, which can be defined as the supremum of SE to fit an image, as in (1), where $I=\mathbb{Z} 2 \rightarrow$ $(0,1, \ldots, 2 n-1)$ is an $n$ bit greyscale image and $B$ is an SE with origin $(i, j)$.

$$
\varphi_{B} I=\bigvee\left\{B_{(i, j)} \mid B_{(i, j)} \leq I\right\}
$$

Conceptually, the opening of an image can be thought of as asking the question, "Does the SE fit inside the object/image foreground?" [18]. The foreground is a set of all features we wish to analyse, so for analysis of an alpha/beta microstructure with platelets in the alpha phase our foreground is the set of all pixels belonging to the alpha phase. If a SE is completely contained within the foreground then the opening will not have any effect, however, if the SE cannot be contained the foreground at a given location then that feature will be removed from the result. This allows us to measure features based on what SE is required to remove them. A simple example of this is shown in Fig. 1, where an image containing three globular features of diameter 20, 50, and 60 are opened using a circular SE of radius 30. Similarly, Borocco's feature extraction method [11] is illustrated in Fig. 2 which shows a linear SE of the same length at 3 different orientations where only one fits. When performing an opening with these SE only the fitting SE would not remove the feature and, therefore, we know its orientation matches that of the SE.

While effective for extracting particular measurements there are clear limitations to varying only a single property of the SE such as radius [28] or orientation [11]. In addition to requiring prior optimisation and parameters and limiting which properties can be measured, this can also cause errors due to incorrectly inferring why the SE does or does not fit a feature. For example, if a linear SE was shorter than the width of a feature it would not fit at any orientation and provide an erroneous measurement.

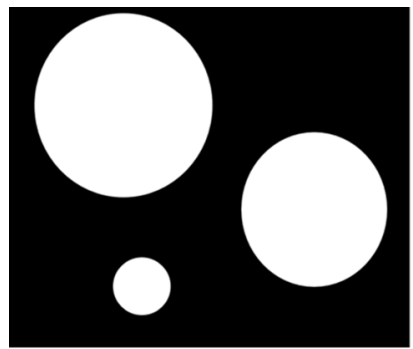

(a)

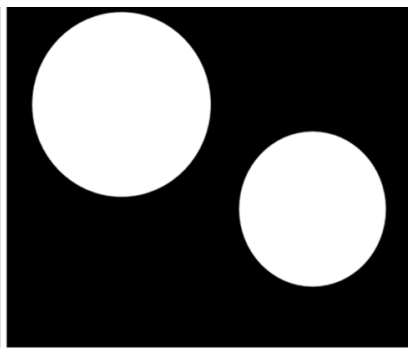

(b)
Figure 1 Illustration of an opening by circular SE where $\mathbf{a}$ is an image with circles of diameter 20,50,60, and $\mathbf{b}$ is the image opened by a circular SE of diameter 30 . 


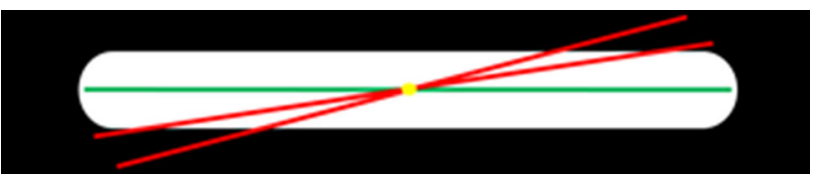

Figure 2 Illustration of rotating SE [11] where all SE pass through the same origin (marked in yellow), the red lines show SE that do not fit due to their orientation so would remove the feature and the green line shows the orientation fits and would retain the feature.

\section{Elongated platelet measurement}

A three-step method is used to overcoming common limitations with morphological measurements and provide an extensive and robust analysis procedure for platelets. Firstly, openings with structuring elements of variable length and orientation are used to extract measurements of the image. Secondly, this data are used to create a detailed orientation space. Finally, computations in the orientation space are demonstrates that produce measurements of key microstructural features.

\section{Measurement from variable length rotating segments}

A morphological feature extraction technique is presented that extends the method of Borocco et al. [11] to include a bivariate optimization for simultaneous orientation and size measurements. The definition provided is also suitable for greyscale images, as is common in microstructural data. As orientation measurements are now based on an optimally fitting length of segment, variations in platelet morphology are less problematic than in the original work [11]. While measurement are per-pixel, the use of the morphological opening ensures measurements within the same platelet, which is important for further analysis. Consider an image containing bright platelets on a dark background. In the case of dark platelets in a bright background, the image should be inversed, as in Fig. 3, so that features we want to measure are bright.Morphological openings of the image, as defined in (1), are deployed to probe the image with a wide range of linear segments, i.e. SEs, of different length and orientation. A technique known as the ultimate opening [32] will allow us to find the optimal length and orientation of segments to fit the image at each location, and thus estimate the size and morphology of microstructural features. To

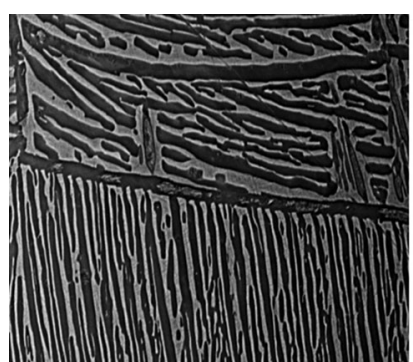

(a)

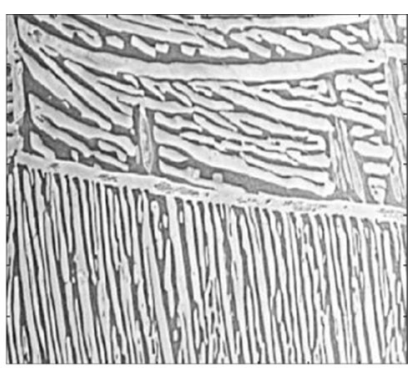

(b)
Figure 3 Example of a microstructural image of alpha platelets where the original image $\mathbf{a}$ has been inverted in $\mathbf{b}$ so the platelets we wish to measure are bright.

construct the ultimate opening, let $\varphi_{\vartheta, \lambda}$ denote an opening by a segment of length $\lambda$ and orientation $\vartheta$. We use the supremum of openings by a rotating structuring element, as in (2). The orientation along which the supremum is found is $\theta=\vartheta$ such that $\varphi_{\vartheta, \lambda}=\varphi_{\lambda}$.

$\varphi_{\lambda}=\bigvee_{\vartheta} \varphi_{\vartheta, \lambda}$

Notice that the supremum of openings verifies the axioms of openings (anti-extensivity, increasingness, idempotence) [18] and it is therefore, itself an opening. The opening removes structures from the image. For the bright platelets in Fig. $2 b$, an opening by any SE that exceeds the perimeters of the structure would return an image where that structure is removed. The residuum $r$ of an opening is defined as what a parameterisable opening, for some given size, removes from the image compared to that of a smaller size, as in (3). The role of the size in our case is played by the length of the segment in the supremum of openings by a rotating segment.

$r_{\lambda}=\varphi_{\lambda-1}-\varphi_{\lambda}$

This is illustrated in Fig. 4, which shows the effect of successive openings and the corresponding residuum.For increasing $\lambda$, the opening will always contain fewer features, however, residum does not always increase as this is relative to what was left after the previous opening, $\varphi_{\lambda-1}$. There exists some length at which the supremum of opening by a rotating segment will entirely remove the platelet. As the opening is an anti-extensive and idempotent operator, the residuum between successive openings will reach a maximum when this occurs. By using the maximum residuum we can ignore smaller, less significant residuum where objects are only partially 


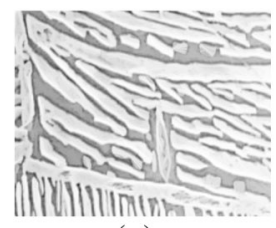

(a)

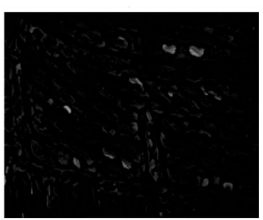

(d)

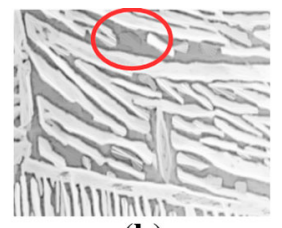

(b)

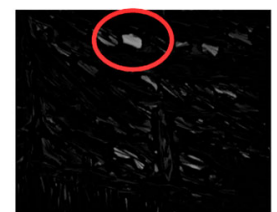

(e)

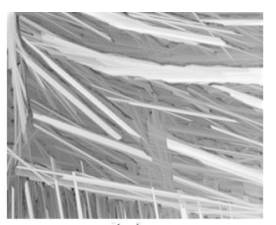

(c)

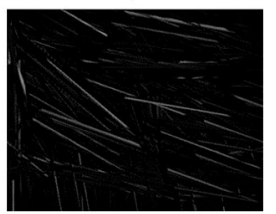

(f)
Figure 4 Illustration of the ultimate opening of a microstructure where $\mathbf{a}$ is $\varphi_{1} \mathbf{b} \varphi_{2}, \mathbf{c} \varphi_{10}, \mathbf{d} r_{1}, \mathbf{e} r_{2}, \mathbf{f} r_{10}$. The red circles indicate the initial removal of a platelet and the corresponding residuum.

removed, as in Fig 4d, and consider the measurement only the SE that removed it most completely. The length of the platelet can, therefore, be given in terms of the size of the ultimate opening, in (4), i.e the length of segment that removes it.

$L=\operatorname{argmax}_{\lambda} r_{\lambda}$

The orientation of the platelet $\theta=\vartheta$ such that $\varphi_{L}=\varphi_{\vartheta, \lambda}$. All measurements from this technique are pixel-wise and thus are per-area measurements. That is they are functions $L=L(x, y)$ and $\theta=\theta(x, y)$. The precision and limits of measurements are determined by the set of lengths and orientations used to perform the openings in (2). This is can be useful either to ignore small secondary features in the image or to balance the runtime of the code against precision.

In the case of platelets that are curvelinear or contain pores, fitting straight elongated structuring elements may lead to some inaccuracy. This is will not often cause significant problems as both width and orientation measurements would remain accurate at the location of measurement. In cases where such features do present a more substantially challenge the ultimate opening in (2) may be replaced by an alternative definition using rank-based morphological filters [33], as shown in (5), where $B$ is an SE of variable length and orientation.

$$
\varphi_{B_{\vartheta \lambda}, r}=\bigvee_{\vartheta \lambda}\left\{\varphi_{B_{\vartheta \lambda}} \mid B_{\vartheta \lambda} \subseteq B \wedge \operatorname{card}\left(B_{\vartheta \lambda}\right)=r\right\}
$$

This definition adds a parameter, $r$, to provide tolerance where $B-r$ is the size of gap permissible in features when fitting the SE. This tolerance can be set at 0 when not required and should always be set such that the tolerance is less than the spacing between platelets, thus SE can be fit such as to ignore pores and small bends in the platelet but without fitting across multiple platelets. This approach has been demonstrated effectively on highly curved structures [34].

\section{Feature length orientation space}

The bi-variate optimization of length and orientation requires the computation of multiple openings that are not all captured in the definition in (4). The FLOS provides a method to fully exploit all of the extracted feature information. The concept of an orientation space, first introduced by Chen and Hsu [35], is a 3dimensional representation of an image where an orientation axis is added to the traditional $(x, y)$ positional axes. Such spaces have an effectively high signal to noise ratio for linear platelets, as these cluster in single plane while noise is widely distributed. However, the traditional orientation space is binary and provides a relatively sparse representation of the image. We instead propose a more detailed feature space capturing the length of features (FLOS) at each position and orientation, rather than a simple binary response. The ultimate opening described in (4) is used to find optimal length values at every orientation, as needed for this space. Rather than using the supremum of openings $\varphi_{\lambda}$, as in (2), we instead use the data from every opening $\vartheta_{\theta, \lambda}$ when calculating the residuum in (3), to produce a residuum that is dependent on both orientation and length. The length at which the maximum residuum occurs for each given $\vartheta$ is the dimension of the platelet in that orientation thus populates the FLOS, as in (6), which is a function $R=R(x, y, \vartheta)$.

$R=\operatorname{argmax}_{\lambda} r_{\lambda, \vartheta}$

The FLOS is illustrated in Fig. 5 which shows the a plot of $R$ at for a single $x, y$ location with and elongated and globular grain. The distinct differences between these profiles enables segmentation of different grain morphologies and facilities measurements,as described in Section III C. As each profile contains multiple datapoints, FLOS based measurements can be robust to small variations and noise. 


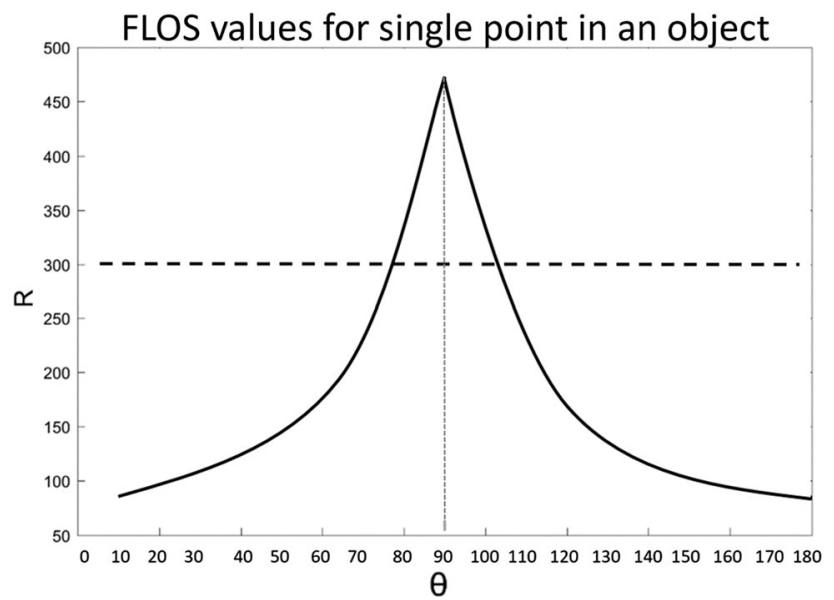

Figure 5 Plot of values in the orientation domain where the solid line shows values for a single point within a platelet and the dashed line shows values for a single point in a globular grain.

\section{Microstructural measurements \\ from the feature length orientation space}

Measurements of key microstructural features such as platelet width, orientation, volume of globular alpha and colony size can all be extracted from the descriptive measurements stored in the FLOS, defined in (6). By default measurement are area-wise but can be normalised if required, since a 100 pixel long object will return that measurement for 100 pixels while a 10 pixel object only returns that measurement 10 times. Consider Fig. 5 which shows plots of the variation of $\mathrm{R}$ in the orientation domain for a platelet and globular grain. The length of each platelet, $f_{l}$, is determined by the maximum value in the orientation domain for each location $(x, y)$ in the image, as in (7). The orientation of platelets, $f_{\theta}$, is the location in the orientation domain where this maximum occurs, as in (8). These measurements are the equivalent of those found using the ultimate opening in (4).

$f_{l}(x, y)=\max _{\theta} R(x, y, \theta)$

$f_{\theta}(x, y)=\operatorname{argmax}_{\theta} R(x, y, \theta)$

Additionally, the FLOS also allows width and shape to be analysed. As platelets are approximately rectilinear, the width of each platelet, $f_{w}$, is equal to the length of the longest SE to fit the platelet at every orientation. This is the minimum value in the orientation domain for each $x, y$ position in $R$ as in (9). As this technique produces pixel-wise measurements, the result will vary throughout the platelet if it narrows at particular regions, giving highly precise measurements.

$f_{w}(x, y)=\min _{\theta} R(x, y, \theta)$

The shape of grains is typically reported by the globular volume fraction we is assessed using the shape profile, as demonstrated in Fig. 5 . The variance in the orientation domain, as in (10) provides a measure of the roundness of image features, $R d$, and thus the probability of being globular. Variance is normalised by dividing the result by the maximum value of $R_{\theta}$, for each pixel, to account for difference in grain size. Alternatively, the ratio between maximum and minimum values can be used as this directly measures the aspect ratio of features, $A R$, as in (11). This is a more intuitively understood measure of grain globularity but would be more sensitive to noise than a variance measurement as it considers only 2 data points.

$$
\begin{aligned}
& R d(x, y)=\max _{\theta} R(x, y, \theta) \\
& A R(x, y)=\operatorname{argmax}_{\theta} R(x, y, \theta)
\end{aligned}
$$

To find colonies using the FLOS, it is recommended to reduce the information to give a sparser orientation space, that indicates the probability of adjacent pixels belonging to the same colony. A binary orientation space, similar to the one used in [11], can be created by detecting local maxima in the FLOS such that only the orientation index containing the maximum length for each pixel is marked as "on." Alternatively, the FLOS also allows this method to be adapted to allow multiple orientations to be switched "on" where the position of the local maxima is unclear. The subsequent grouping algorithm can then use that data to decide which colony this area belongs to, based on orientation and spatial location relative to other platelets. Different grouped algorithms are possible and can factor in expert knowledge of the microstructure under study, and include tolerances for colonies where platelets are not perfectly parallel. In Algorithm 1, we present one such method where similarly orientated features are grouped based on the distance between them, $w$, angular tolerance $T$ and the minimum colony size to identify for as a percentage of the largest colony found, cs. The parameter $w$ determines how close platelets need to be within the colony, while $T$ determines the difference in orientation permitted for platelets to be considered to belong to the same colony. As the FLOS 
measures orientation at discrete angles there is also a natural tolerance to misaligned platelets based on $S_{\theta}$.

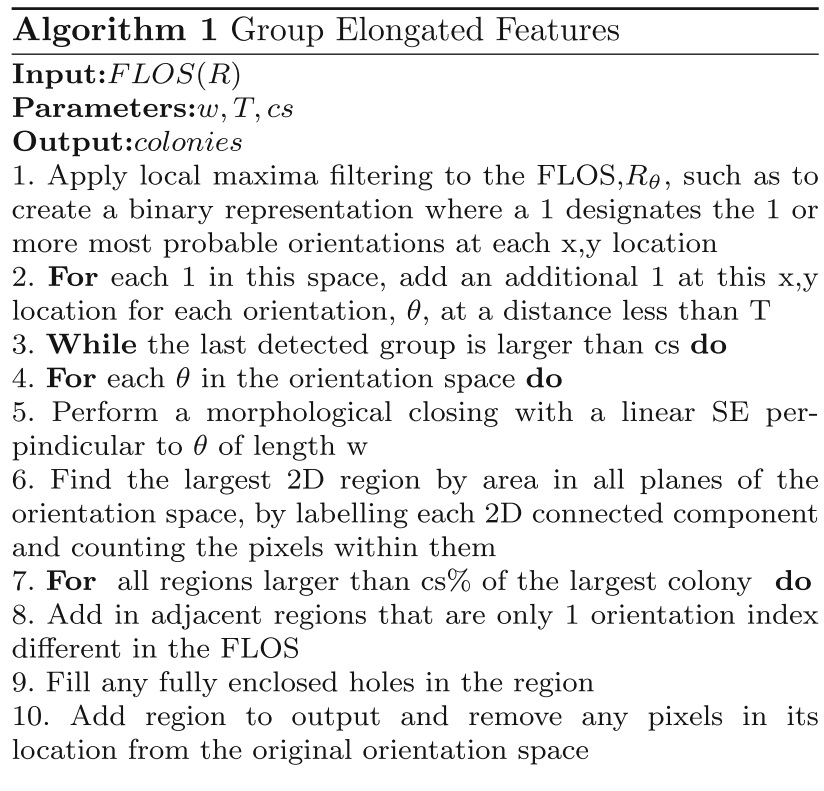

The benefit of using the FLOS, and such an algorithm, for a grouping platelets into colonies is apparent if we consider the microstructure in Fig. 6. This microstructure contains thin platelets at different angles, globular grains, and has poorly delineated boundaries. Figure $6 \mathrm{~b}$ shows a likely result from many software segmentation methods due to the lack of visible boundaries. However, FLOS orientation data, shown by the colormap in Fig. 6c, shows an obvious relationship between platelets of the same colony. Applying Algorithm 1 to this data allows for successful colony segmentation, as shown in Fig. 6d, which there is no obvious way to compute from the original greyscale values.

\section{Results}

The performance of our new technique is demonstrated by applying it to analyse elongated grains of complex Ti-6Al-4V microstructures. This is a widely used material that produces challenging microstructures where image processing has had limited success due to factors such as, the low resolution of image features [4], inconsistent feature shape and dimensions, [7] and large variations between images [26], as in Fig. 7. Two different datasets are used which combined provide a wide range of

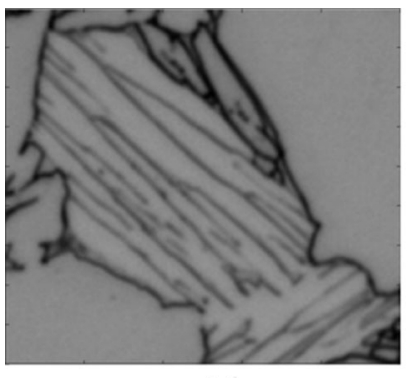

(a)

(c)

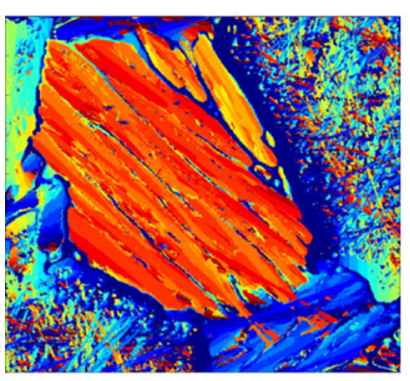

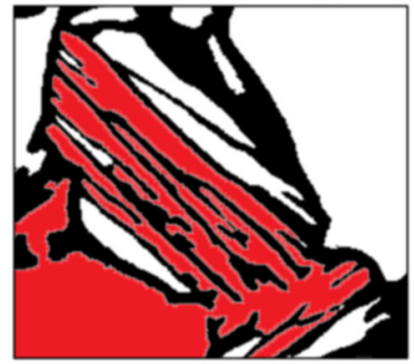

(b)

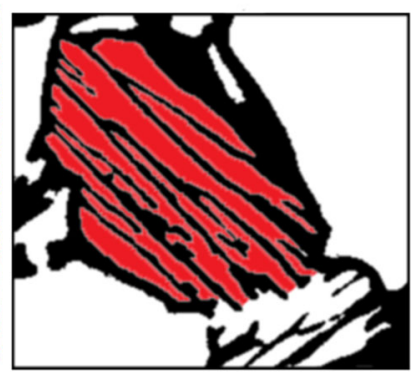

(d)
Figure 6 FLOS benefit where a original image, b largest connected alpha phase region, c colormap of our orientation measurements and $\mathbf{d}$ largest colony from Algorithm 1.

microstructure morphology from different sources, thermal-mechanical processes, and imaging setups. These include images from scanning electron microscope (SEM) and optical microscope (OM) technology, which image similar features but with different noise levels and contrast, so help demonstrate the robustness of the technique. The first dataset, Dataset A, is an original dataset used in this work, while Dataset B was made publicly available by other authors [27]. Dataset A curates images from different experimental and imaging setups to reflect, as much as possible, the variations in real world microscopy. Dataset $B$ has less variation but are more challenging to analyse. 30 different microstructures (14 from A, 16 from B) were analysed using the FLOS and compared to ground truth measurements produced using international standards [1, 2]. Microstructures from Dataset A are labelled A1-A14 and those from Dataset B denoted B1-B16. In Dataset A, multiple images of each microstructure were used if required to ensure statistically valid measurements under these standards. Images ranging in size up to a maximum of $2000 \times 2000$ pixels with a scanned area of up to $234 \mathrm{~mm}^{2}$ per sample. Implemented on a modern laptop with an i7 processor, the FLOS techniques take from $20 \mathrm{~s}$ to $3 \mathrm{~min}$ to measure every 


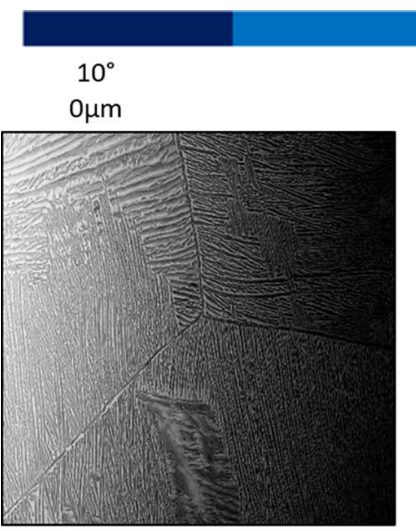

(a)

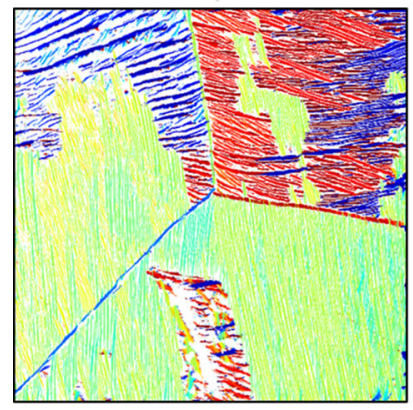

(e)

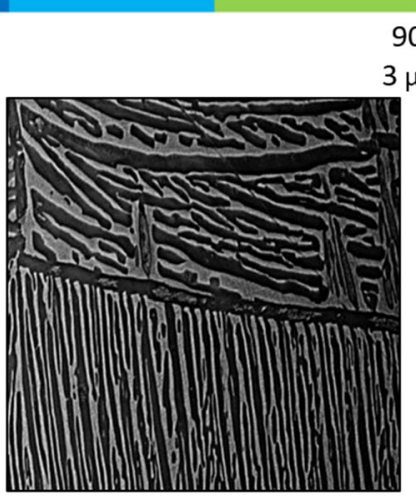

(b)

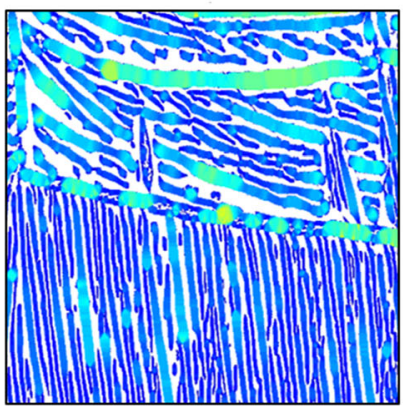

(f) $90^{\circ}$

$3 \mu \mathrm{m}$

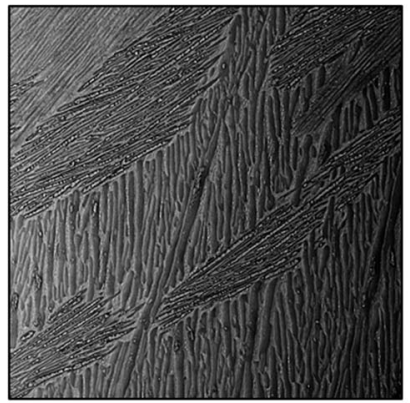

(c)

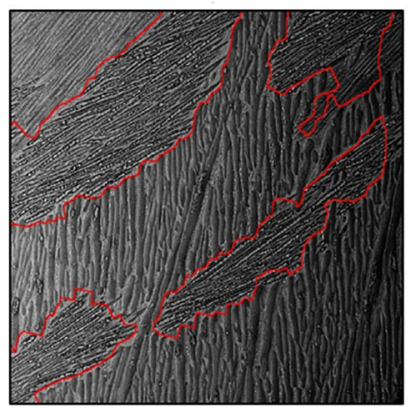

(g)

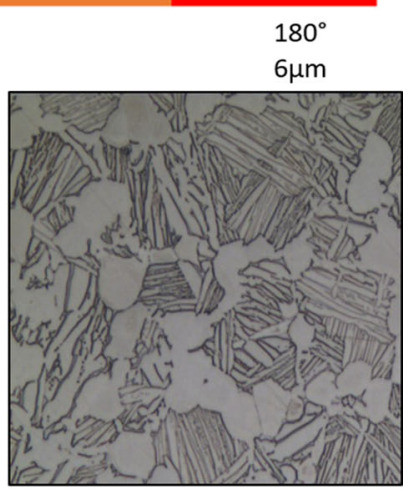

(d)

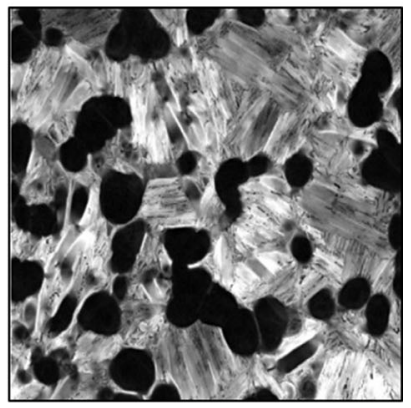

(h)

Figure 7 Illustration of images from the dataset and measurement maps where a-c shows microstructure of platelets only imaged by an SEM, d shows a microstructure with both globular alpha and platelets imaged by an OM, e shows an orientation map of $\mathbf{a}, \mathbf{f}$

property depending on image size, while existing standards required at least $15 \mathrm{~min}$ to measure only platelet width. Along with the repeatability and lack of manual intervention this gives the new method a significant advantage, however, this was achieved using robust parameters, specifically; $S_{\theta}=10^{\circ}, S_{L}=2$ pixels and $L_{\max }=91$. Application specific optimisations could reduce measurement time further, as could more efficient GPU implementations of morphological operators [36], that were outside the scope of this study.

A key benefit of the FLOS technique is that it can be applied directly to greyscale images without prior thresholding and phase separation. However, methods of extracting a single phase of materials are widely used and often beneficial to constrain measurements to a particular subset of the microstructure [4]. To demonstrate the applicability of the FLOS to both cases phase seperation methods from [4] are used when analysing Dataset A, while for Dataset B the original greyscale images are used.

show a width map of $\mathbf{b}, \mathbf{g}$ shows segmented colonies from $\mathbf{c}, \mathbf{h}$ shows and elongation map of $\mathbf{d}$, i.e. the probability of pixel being a platelet and not globular.

Variations in measurements between users of $\pm 16 \%$ are expected for size measurements and $10 \%$ for volume fraction measurements [1, 2]. In line with previous work in this area we accept results within this range to represent valid microstructural measurements, as an absolute ground truth is not known for this type of microstructural analysis [4]. While the FLOS can measure platelet width, orientation, globular volume fraction and colony size, it is not possible to reliably measure each of these properties in all microstructures in these datasets. For this reason, the subsequent evaluations will omit any microstructures for which valid measurement are impossible using existing standards.

An analysis of platelet orientation and widthis shown in Table 1. For each microstructure, the modal orientation and mean width of platelets are measured. The inherit filtering capabilities of the FLOS are exploited to constrain measurements only to platelets. That is a threshold is set on the shape descriptor in Eq. (10) such that any pixel within a 
globular feature is omitted from measurement. It can be observed from Table 1 that the measurements achieved for both platelet width and platelet orientation closely agree with what was achieved by existing standards. However, while existing standards require two separate measurement techniques, the FLOS measures both in a single, repeatable operation with no human intervention. No clear difference in performance can be observed between datasets, demonstrating that pre-processing and phase separation is not unnecessary with this technique. Only a few microstructures, such as B10, Fig. 8, shows disparity in width measurement outside the expected variability of existing standards. This is caused by the relatively low resolution of these platelets, as these errors as still less then 4 pixels, and very poor delineation of boundaries. This is supported by high accuracy of platelet orientation measurement for these same images, which is a less resolution limited measurement and also does not require boundaries be as clear at all locations.

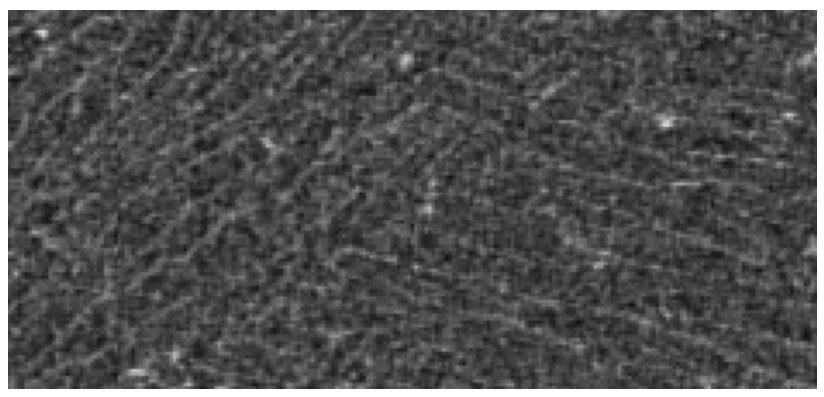

Figure 8 Crop from image 10 showing poor boundary delineation.

These results also illustrate how discriminative image processing techniques can remain relevant and compliment emerging deep learning technologies. The microstructures in Dataset $\mathrm{B}$ are publishing alongside a very recent paper using deep learning for microstructure classification [27]. However, the original paper then post-processes each microstructure depending on the result, as these networks are not always ideally suited for this task. The measurement techniques used were similar to that in [4], which do
Table 1 Platelet width and orientation

\begin{tabular}{|c|c|c|c|c|c|c|}
\hline \multirow[t]{2}{*}{ Microstructure } & \multicolumn{3}{|c|}{ Platelet width } & \multicolumn{3}{|c|}{ Platelet orientation $\left({ }^{\circ}\right)$} \\
\hline & FLOS & GT & Difference & (FLOS) & (GT) & (Difference) \\
\hline A1 & $2.58 \mu \mathrm{m}$ & $2.19 \mu \mathrm{m}$ & $+0.39 \mu \mathrm{m}$ & 100 & 100 & 0 \\
\hline A2 & $2.17 \mu \mathrm{m}$ & $2.15 \mu \mathrm{m}$ & $+0.02 \mu \mathrm{m}$ & 90 & 90 & 0 \\
\hline A3 & $2.65 \mu \mathrm{m}$ & $2.86 \mu \mathrm{m}$ & $-0.21 \mu \mathrm{m}$ & 90 & 90 & 0 \\
\hline A4 & $2.09 \mu \mathrm{m}$ & $2.22 \mu \mathrm{m}$ & $-0.13 \mu \mathrm{m}$ & 80 & 90 & 10 \\
\hline A5 & $1.73 \mu \mathrm{m}$ & $1.93 \mu \mathrm{m}$ & $-0.20 \mu \mathrm{m}$ & 40 & 45 & 5 \\
\hline A6 & $2.77 \mu \mathrm{m}$ & $2.41 \mu \mathrm{m}$ & $-0.36 \mu \mathrm{m}$ & 30 & 30 & 0 \\
\hline A7 & $2.40 \mu \mathrm{m}$ & $2.25 \mu \mathrm{m}$ & $+0.15 \mu \mathrm{m}$ & $\times$ & $\times$ & $x$ \\
\hline A8 & $2.46 \mu \mathrm{m}$ & $2.38 \mu \mathrm{m}$ & $+0.08 \mu \mathrm{m}$ & $x$ & $x$ & $\times$ \\
\hline B1 & $27.12 \mathrm{px}$ & $27.61 \mathrm{px}$ & $-0.49 \mathrm{px}$ & 160 & 165 & -5 \\
\hline B2 & $22.40 \mathrm{px}$ & $22.02 \mathrm{px}$ & $+0.38 \mathrm{px}$ & 100 & 90 & 10 \\
\hline B3 & $22.69 \mathrm{px}$ & $19.66 \mathrm{px}$ & $+3.03 \mathrm{px}$ & 170 & 170 & 0 \\
\hline B4 & $22.10 \mathrm{px}$ & $20.64 \mathrm{px}$ & $+1.46 \mathrm{px}$ & 100 & 100 & 0 \\
\hline B5 & $23.65 \mathrm{px}$ & $22.50 \mathrm{px}$ & $+1.15 \mathrm{px}$ & 10 & 10 & 0 \\
\hline B6 & $24.50 \mathrm{px}$ & $23.77 \mathrm{px}$ & $+0.73 \mathrm{px}$ & 10 & 10 & 0 \\
\hline B7 & $26.81 \mathrm{px}$ & $26.66 \mathrm{px}$ & $+0.15 \mathrm{px}$ & 40 & 45 & -5 \\
\hline B8 & $26.85 \mathrm{px}$ & $25.66 \mathrm{px}$ & $+1.19 \mathrm{px}$ & 40 & 45 & -5 \\
\hline B9 & $13.87 \mathrm{px}$ & $11.25 \mathrm{px}$ & $+2.62 \mathrm{px}$ & 80 & 85 & -5 \\
\hline B10 & $14.01 \mathrm{px}$ & $10.44 \mathrm{px}$ & $+3.68 \mathrm{px}$ & 160 & 165 & -5 \\
\hline B11 & $13.79 \mathrm{px}$ & $12.60 \mathrm{px}$ & $+1.19 \mathrm{px}$ & 80 & 80 & 0 \\
\hline B12 & $19.80 \mathrm{px}$ & $17.41 \mathrm{px}$ & $+2.39 \mathrm{px}$ & 110 & 110 & 0 \\
\hline B13 & $22.42 \mathrm{px}$ & $20.58 \mathrm{px}$ & $+1.84 \mathrm{px}$ & 40 & 40 & 0 \\
\hline B14 & $22.81 \mathrm{px}$ & $22.16 \mathrm{px}$ & $+0.65 \mathrm{px}$ & 70 & 70 & 0 \\
\hline B15 & $26.26 \mathrm{px}$ & $23.16 \mathrm{px}$ & $+3.10 \mathrm{px}$ & 10 & 15 & -5 \\
\hline $\mathrm{B} 16$ & $19.51 \mathrm{px}$ & $17.11 \mathrm{px}$ & $+2.40 \mathrm{px}$ & 60 & 60 & 0 \\
\hline
\end{tabular}


not perform optimally on the images in our current study. The FLOS method could complement this by providing a more detailed analysis technique that could be applied later in the analysis pipeline, i.e. after the network detects platelet microstructures suitable for applying our technique.

The pixel level measurements produced by the FLOS also enable useful colourmaps to be generated, as shown in Fig. 7. Variations in platelet width and orientation are highlighted by the fact these differ in colour from the surrounding platelets making it easier to spot anomalies in the microstructure. This will ultimately allow the use of the FLOS as a qualitative analysis tool that extends beyond the measurements demonstrated in this paper. This is especially useful when variations in brightness obscure the visibility of platelets to the human eye, as in Fig. 7a. If the FLOS is applied directly to the greyscale image, the resulting feature space eliminates these contrast differences and produces colourmaps where all features are clearly visible, as seen in Fig. 7e. Furthermore, these measurement maps would allow for interesting correlations with other measurement and microstructural analysis techniques. For example, chemical elemental mapping could allow precise comparison between chemical composition and microstructure morphology. While such studies are outside the scope of this work, the possibility to do so is an advantage of the FLOS technique, as existing measurement standards do not produce the required mapping and spatial precision of microstructure measurement.

An analysis of globular volume fraction is shown in Table 2. For each microstructure, the percentage of the totally scanned area consisting of globular alpha grains is measured. This was calculated by applying the equation in (10) to achieve an elongation map, as in Fig. $7 \mathrm{~h}$, thresholding to find globular regions, i.e. the darkest pixels in the elongation map, and measuring this remaining area as a percentage of the scanned area. The threshold is set such that only those regions with an aspect ratio above 2:1 are considered to be globular. This threshold was chosen empirically using 1 image and kept consistent for every image in the datasets used in this trial. As this required bi-modal microstructures with both globular grains and platelets visible, only a smaller subset of our dataset could be used, as many microstructures did not contain globular grains.
Table 2 Globular Volume Fraction measurement

\begin{tabular}{llll}
\hline Microstructure & \multicolumn{3}{l}{ Globular volume fraction (\%) } \\
\cline { 2 - 4 } & FLOS & GT & Difference \\
\hline A7 & 75 & 71 & +4 \\
A8 & 77 & 74 & +3 \\
A9 & 34 & 36 & -2 \\
A10 & 29 & 24 & +5 \\
A11 & 28 & 25 & +3 \\
A12 & 38 & 34 & +4 \\
A13 & 19 & 20 & -1 \\
A14 & 52 & 36 & +16 \\
\hline
\end{tabular}

Table 2 shows strong agreement with the ground truth, within accepted tolerances [1]. The exception was in a single microstructural dataset, where very high levels of grain clustering and poor delineation of boundaries created a $16 \%$ error. In such cases, expert materials scientists also struggle to distinguish aligned globular grains from platelets, so greater uncertainty in measurements from the standard is expected. As with other features, an intensity map of globularity, shown in Fig. $7 \mathrm{~h}$, is produced that is useful for further analysis and increasing confidence in the automated measurement. The intensity map shown is normalized using the maximum and minimum values of the image to show black pixels for the most equiaxed features and white pixels for the most elongated. This highlights the spatial distribution of different grain morphologies and indicates, as medium grey areas, regions about which the software is likely to be most uncertain. An analysis of colony size is shown in Table 3. Previous attempts at automated colony measurement struggled due to unreliable orientation measurements [4]. The extensive data contained in the FLOS, combined with the noise reduction benefits of analysing this in an orientation space, allows more accurate identification and measurements of colonies, using Algorithm 1. Distinct colonies of platelets do not exist in all microstructures, so a subset of the datasets are again used. Due to the desire to measurement platelet width, a high percentage of colonies frequently exceed the boundaries of the image. For the purpose of this study both the automated technique and ground truth standards ignore this problem and measure simply what is visible at the colony. This means that this does not 
Table 3 Colony size measurements

\begin{tabular}{llll}
\hline Microstructure & \multicolumn{3}{l}{ Mean colony size $(\mu \mathrm{m})$} \\
\cline { 2 - 4 } & FLOS & GT & Difference \\
\hline A1 & 2.58 & 2.19 & +0.39 \\
A2 & 2.17 & 2.15 & +0.02 \\
A3 & 2.65 & 2.86 & -0.21 \\
A4 & 2.09 & 2.22 & -0.13 \\
A5 & 1.73 & 1.93 & -0.20 \\
A6 & 2.77 & 2.41 & -0.36 \\
A7 & 2.40 & 2.25 & +0.15 \\
A8 & 2.46 & 2.38 & +0.08 \\
\hline
\end{tabular}

accurately describe material properties, but allows fair evaluation of the method proposed in this paper.

Table 3 shows results are mostly within the range expected by existing standards and provides a good illustration of the usefulness of the FLOS to further analysis pipelines. Only microstructure A6, which had the most complex and uncertain structure of platelets, as shown in Fig. 7d, has colony measurements outside the expected variation. A limitation of this method is that the proposed approach considers only the parallel nature of platelets within colonies and not the other factors that might identify them. For example, Fig. 7a shows a triple point towards the center of the image where the boundaries of three colonies meet, which this method is not currently trained to recognise. Future work should adapt Algorithm 1 to identify such features, which should be aided by the orientation information provided from the current method.

\section{Comparative study}

To the best of the authors knowledge no accurate, fully automated method for colony and globular volume fraction measurement has currently been presented and thus the FLOS offers novel functionality as a microstructural analysis tool. Methods to measure platelet width and orientation have been presented, but in different circumstances and with limitations the FLOS can exceed. In this section, we compare the results achieved to what could been achieved with those methods.

We selected a number of suitable approaches for comparison [7, 11, 17, 28]. Unlike the FLOS, these techniques cannot be applied directly to greyscale images and do not contain any shape information, giving these an immediate disadvantage. As such they cannot isolate measurements to platelets only in bi-modal microstructures such as in Fig. 7d. To provide the fairest possible comparison, we select a subset of images of exclusively platelet microstructures from Dataset A for this comparison. Some authors expect inaccuracies from existing methods due to size variance $[7,11]$. As we believe the FLOS should be robust to such problems we also include artificial binary data that will examine specifically the effects of size variance on these methods. Artificial data was created manually to show different degrees of size variation, as in Fig. 9.

Of the relevant existing methods only a recent paper studying graphene structures [11] enables orientation measurement. The method probes the image with a single length of SE set in advance, a limitation compared to our method in which a wide range of SE can be used. Setting the length to low will result in inaccurate measurements, while setting this too high will result in regions not being measured at all. We demonstrate this trade-off by repeating measuring with 2 different SE length parameters. Each parameter is set at the largest length to measure at least $90 \%$ and $75 \%$ of the microstructure respectively. Table 4 provides a statistical description of these results. This demonstrates that the FLOS provides the most consistent results, as it is able to select an optimal SE with which to measure each feature in the image.

Width measurements are more common in the literature. We compare two recent techniques from other domains [17, 28], a classical approach using the distance transform [16] and a titanium specific technique proposed by Tiley and Collins et al. [6, 7]. This latter method measures feature width based on the

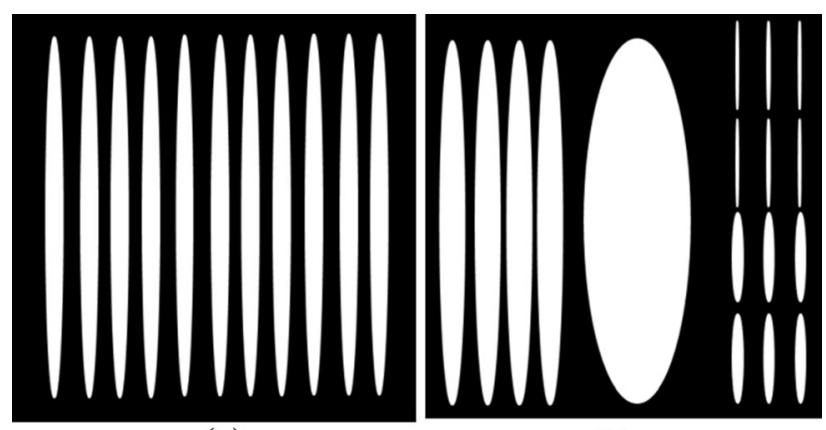

(a)

(b)

Figure 9 Example artificial data where a artificially drawn platelets of consistent dimensions and $\mathbf{b}$ artificially drawn platelets of variable dimensions. 
Table 4 Comparison of measurement error in modal orientation measurements, where error is the difference between automated measurement and the ground truth

\begin{tabular}{llll}
\hline & FLOS & \multicolumn{2}{l}{ Fixed SE [11] } \\
\cline { 3 - 4 } & & $90 \%$ measured & $75 \%$ measured \\
\hline Mean error & $0^{\circ}$ & $20^{\circ}$ & $9^{\circ}$ \\
Maximum error & $0^{\circ}$ & $80^{\circ}$ & $50^{\circ}$ \\
Minimum error & $0^{\circ}$ & $0^{\circ}$ & $0^{\circ}$ \\
Datasets within tolerance & $100 \%$ & $64 \%$ & $82 \%$ \\
\hline
\end{tabular}

intercept length of randomly oriented line segments so is conceptually similar to our work. However, an important caveat is that in the original work the authors describe this concept only to estimate 3D properties of material. For this study we make a simple modification for the 2D slices by using only the mean intercept length, and removing the additional 3D estimation. This comparison therefore represents the applicability of this concept to measurement of 2D images, but does not represent the quality or claims in the original paper. Nevertheless, we include this comparison as the similarity in how measurements are extracted help to highlight the specific benefits of using an orientation space.

Table 5 shows a statistical comparison of measurement differences from each technique, where measurements are provided in pixels so that magnification level does not bias the result. The FLOS achieved more consistent results overall, with other methods achieving strong results on certain data. This demonstrates the robustness of the FLOS, as seen in the previous experiment. Figure 10 highlights this by plotting measurement error against the ground truth for each dataset, where the artificial datasets are marker AR1-AR5 and real microstructures are marked Ti1-Ti6.

Collins et al. [7] expect a drop in accuracy for aspect ratios above 20:1, which, given AR4 and AR5

Table 5 Comparison of mean width measurement error in pixels defined as the difference between automated measurements and the ground truth

\begin{tabular}{llllll}
\hline & \multicolumn{5}{l}{ Width measurements $(\mathrm{px})$} \\
\cline { 2 - 6 } & FLOS & {$[27]$} & {$[17]$} & {$[7,11]$} & {$[16]$} \\
\hline Mean error & 1 & 3 & 12 & 19 & 4 \\
Maximum error & 2 & 10 & 42 & 120 & 14 \\
Minimum error & 0 & 1 & 1 & 0 & 0 \\
Samples within tolerance & $100 \%$ & $73 \%$ & $45 \%$ & $27 \%$ & $73 \%$ \\
\hline
\end{tabular}

have aspect ratios below this threshold, appears to explain some errors. For other techniques it is the inherent filtering abilities and resilience to noise of the FLOS that causes the improved accuracy. While microstructures with globular particles were ommited here, noise and artefacts often have a rounder shape than platelets and thus the FLOS is better at discounting these. Dents, dust particles or pores may all be filtered out from platelet measurements where they do not match the expected shape. Thus by measuring multiple properties simultaneously, this type of filtering allows the FLOS to outperform other methods based on similar principles, such as [28].

\section{Summary}

A fully automated image processing technique has been presented for quantitatively analysing microstructures containing platelets. Measurements of platelet width, orientation, globular volume fraction and colony size are all made possible by this technique. These measurements are all achieved in a per area basis, allowing for both accurate statistical measurements and effective property maps to aid further microstructural investigations. The proposed FLOS technique was successfully applied to both

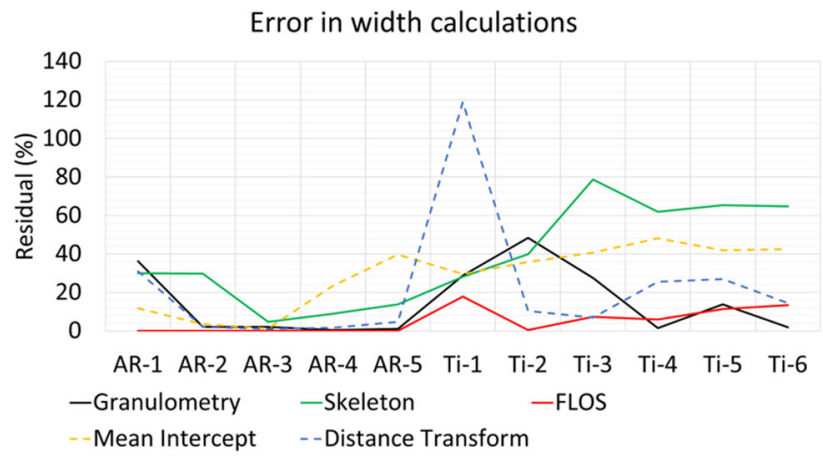

Figure 10 Graph of measurement error for each method as described by the \%difference between the ground truth and the automated measurement. 
greyscale images and binary (phase segmented) images with equally accurate results. By using both novel and public datasets the robustness of the technique to a wide variety of microstructures is demonstrated. Comparison with existing image processing methods from other domains demonstrate that the FLOS has unique advantages in terms of the extent of measurement and reliability when encountering variations in both noise and microstructural morphology. FLOS generated results align with existing ASTM standards and the automated nature of the solution eliminates user bias and subjectivity, and ensures repeatability. The run-time of the software is much faster than applying these standards, which offers great benefits in both analysis cost and scalability. In addition to the quantitative measures, the per-pixel measurements provide enables the generation of useful colourmaps of each property. This can increase user confidence in results and aid further analysis. Future work is expected to be able to further optimise this technique to reduce measurement time, as well investigate the use of the vast quantity of data stored within the FLOS to identify scratches, cracks and other defects.

\section{Acknowledgements}

This work was supported by Engineering and Physical Sciences Research Council through grant EP/ I015698/1.

Open Access This article is licensed under a Creative Commons Attribution 4.0 International License, which permits use, sharing, adaptation, distribution and reproduction in any medium or format, as long as you give appropriate credit to the original author(s) and the source, provide a link to the Creative Commons licence, and indicate if changes were made. The images or other third party material in this article are included in the article's Creative Commons licence, unless indicated otherwise in a credit line to the material. If material is not included in the article's Creative Commons licence and your intended use is not permitted by statutory regulation or exceeds the permitted use, you will need to obtain permission directly from the copyright holder. To view a copy of this licence, visit http://creativecommons.org/licen ses/by $/ 4.0 /$.

\section{Data availability statement}

A version of this software is available, alongside ground truth measurements for the public dataset, at https:/ / doi.org/10.15129/4d3be392-2284-4f34-9ebde6ad205c2641. The authors certify that they have NO affiliations with or involvement in any organization or entity with any financial interest (such as honoraria; educational grants; participation in speakers' bureaus; membership, employment, consultancies, stock ownership, or other equity interest; and expert testimony or patent-licensing arrangements), or nonfinancial interest (such as personal or professional relationships, affiliations, knowledge or beliefs) in the subject matter or materials discussed in this manuscript.

\section{References}

[1] ASTM. E112 Standard test method for determining average grain size

[2] ASTM. E562 Standard test method for determining volume fraction by systematic manual point cloud

[3] Lutjering G, Williams JC (2007) Titanium, 2nd edn. Springer, Berlin

[4] Campbell A, Murray P, Yakushina E, Marshall S, Ion W (2018) New methods for automatic quantification of microstructural features using digital image processing. Mater Des 141:395-406. https://doi.org/10.1016/J.MATDE S.2017.12.049

[5] Semiatin SL, Knisley SL, Fagin PN, Barker DR, Zhang F (2003) Microstructure evolution during alpha-beta heat treatment of Ti-6Al-4V. Metall Mater Trans A 34(10):2377-2386. https://doi.org/10.1007/s11661-003-030 $0-0$

[6] Tiley J, Searles T, Lee E, Kar S, Banerjee R (2004) Quantification of microstructural features in $\alpha / \beta$ titanium alloys. Mater Sci 372(1-2):191-198. http://www.sciencedirect.com/ science/article/pii/S0921509303014758. Accessed 31 Aug 2017

[7] Collins P, Welk B, Searles T, Tiley J, Russ J (2009) Development of methods for the quantification of microstructural features in $\alpha+\beta$-processed $\alpha / \beta$ titanium alloys. Mater Sci 508(1-2):174-182. http://www.sciencedirect.com/science/ar ticle/pii/S0921509308014937. Accessed 31 Aug 2017

[8] Merveille O, Talbot H, Najman L, Passat N (2017) Curvilinear structure analysis by ranking the orientation responses of path operators. IEEE Trans Pattern Anal Mach Intell 40(2):304-317 
[9] Moreno R, Smedby Ö (2015) Gradient-based enhancement of tubular structures in medical images. Med Image Anal 26(1):19-29. https://doi.org/10.1016/j.media.2015.07.001

[10] Jeon B, Jang Y, Shim H, Chang H-J (2019) Identification of coronary arteries in CT images by Bayesian analysis of geometric relations among anatomical landmarks. Pattern Recognit 96:106958. https://doi.org/10.1016/j.patcog.2019. 07.003

[11] Borocco A, Fellah C, Braun J, Berger M-H, Dokládal P (2017) Morphological characterization of graphene plans stacking, pp 435-446

[12] Liu L, Liu Y, Zhang J (2018) Learning-based hand motion capture and understanding in assembly process. IEEE Trans Ind Electron 66(12):9703-9712

[13] Ballard D (1981) Generalizing the Hough transform to detect arbitrary shapes. Pattern Recognit 13(2):111-112. https://w ww.sciencedirect.com/science/article/pii/B97800805158165 00696. Accessed 29 May 2018

[14] Freeman WT, Adelson EH (1991) The design and use of steerable filters. IEEE Trans Pattern Anal Mach Intell 13(9):891-906

[15] Mukhopadhyay P, Chaudhuri BB (2015) A survey of Hough transform. Pattern Recognit 48(3):993-1010. https://doi.org/ 10.1016/j.patcog.2014.08.027

[16] Russ JC (2016) The Image processing handbook

[17] Adhikari RS, Moselhi O, Bagchi A (2014) Image-based retrieval of concrete crack properties for bridge inspection. Autom Constr 39:180-194. https://doi.org/10.1016/J.AUT CON.2013.06.011

[18] Soille P (2013) Morphological image analysis: principles and applications. Springer, Berlin

[19] Xing F, Yang L (2016) Robust nucleus/cell detection and segmentation in digital pathology and microscopy images: a comprehensive review. IEEE Rev Biomed Eng 9:234-263

[20] Nellros F, Thurley MJ, Jonsson H, Andersson C, Forsmo SPE (2015) Automated measurement of sintering degree in optical microscopy through image analysis of particle joins. Pattern Recognit 48(11):3451-3465. https://doi.org/10.1016/ j.patcog.2015.05.012

[21] $\mathrm{Gu} \mathrm{J}$ et al (2018) Recent advances in convolutional neural networks. Pattern Recognit 77:354-377. https://doi.org/10. 1016/j.patcog.2017.10.013

[22] Sassi P, Tripicchio P, Avizzano CA (2019) A smart monitoring system for automatic welding defect detection. IEEE Trans Ind Electron 66(12):9641-9650

[23] DeCost BL, Francis T, Holm EA (2018) High throughput quantitative metallography for complex microstructures using deep learning: a case study in ultrahigh carbon steel. a rXiv:1805.08693. Accessed 5 Feb 2019
[24] de Albuquerque VHC, Cortez PC, de Alexandria AR, Tavares JMRS (2008) A new solution for automatic microstructures analysis from images based on a backpropagation artificial neural network. Nondestruct Test Eval 23(4):273-283. https://doi.org/10.1080/ 10589750802258986

[25] Litjens G et al (2017) A survey on deep learning in medical image analysis. Med Image Anal 42:60-88. https://doi.org/ 10.1016/J.MEDIA.2017.07.005

[26] Dimiduk DM, Holm EA, Niezgoda SR (2018) Perspectives on the impact of machine learning, deep learning, and artificial intelligence on materials, processes, and structures engineering. Integr Mater Manuf Innov 7(3):157-172. http s://doi.org/10.1007/s40192-018-0117-8

[27] Baskaran A, Kane G, Biggs K, Hull R, Lewis D (2020) Adaptive characterization of microstructure dataset using a two stage machine learning approach. Comput Mater Sci. h ttps://doi.org/10.1016/j.commatsci.2020.109593

[28] Statella T, Pina P, da Silva EA (2016) Automated width measurements of Martian dust devil tracks. Aeolian Res 20:1-6. https://doi.org/10.1016/J.AEOLIA.2015.11.001

[29] Rivest J-F (1993) Morphological gradients. J Electron Imaging 2(4):326. https://doi.org/10.1117/12.159642

[30] Matheron G (1975) Random sets and integral geometry. Probab Math Stat 385969(52):6828. http://www.ams.org/jo urnals/bull/1975-81-05/S0002-9904-1975-13853-5/. Accessed 29 Jan 2018

[31] Serra J (1986) Introduction to mathematical morphology. Comput Vis Graph Image Process 35(3):283-305. http://w ww.sciencedirect.com/science/article/pii/0734189X8690002 2. Accessed 7 Sep 2017

[32] S B-I, V Computing and undefined 2007. Numerical residues. Elsevier. https://www.sciencedirect.com/science/articl e/pii/S0262885606002563. Accessed 17 Aug 2018

[33] Soille P, Talbot H (2001) Directional morphological filtering. IEEE Trans Pattern Anal Mach Intell 23(11):1313-1329

[34] Morard V, Dokládal P, Decenciere E (2014) Parsimonious path openings and closings. IEEE Trans Image Process 23(4):1543-1555

[35] Chen Y-S, Hsu W-H (1989) An interpretive model of line continuation in human visual perception. Pattern Recognit 22(5):619-639. https://doi.org/10.1016/0031-3203(89)9003 $0-7$

[36] Karas P et al (2015) GPU implementation of linear morphological openings with arbitrary angle. J Real-Time Image Process 10(1):27-41

Publisher's Note Springer Nature remains neutral with regard to jurisdictional claims in published maps and institutional affiliations. 